\title{
FATORES CONTEXTUAIS DE IMPORTÂNCIA NA MELHORIA DO DESEMPENHO NA AGRICULTURA BRASILEIRA: UMA ANÁLISE COM BASE EM MODELOS DEA
}

Geraldo da Silva e Souza

Eliane Gonçalves Gomes

Eliseu Roberto de Andrade Alves

Empresa Brasileira de Pesquisa Agropecuária (Embrapa)

PqEB, Av. W3 Norte final, 70770-901, Brasília, DF, Brasil

\{geraldo.souza; eliane.gomes; eliseu.alves\}@embrapa.br

Resumo: Utiliza-se uma fronteira de produção do tipo não paramétrica na avaliação da produção agrícola dos municípios brasileiros. Com o uso de métodos de regressão fracionária, caracteriza-se a intensidade da associação entre eficiência técnica de produção e construtos contextuais representando medidas que refletem o direcionamento da pesquisa da Embrapa, a assistência técnica ao meio rural e itens típicos de formas de entrega de políticas públicas. Conclui-se que esses, na realidade, são os únicos estatisticamente significantes para a melhoria da eficiência de produção no meio rural brasileiro.

Palavras-chave: DEA, Regressão fracionada, Covariáveis, Agricultura.

\begin{abstract}
We use a non-parametric production frontier to evaluate the agricultural production of the Brazilian counties. By means of fractional regression model, we characterize the intensity of the association between technical efficiency and contextual constructs representing measures that reflect the direction of Embrapa's research, rural technical assistance and typical features of public policies. We conclude that these, in fact, are the only statistically significant effects for the improvement of production efficiency in the Brazilian countryside.
\end{abstract}

Key-words: DEA, Fractional regression, Covariates, Agriculture. 


\section{INTRODUÇÃO:}

Em estudo recente sobre agricultura brasileira com base nos Censos Agropecuários de 1995/96 e 2006, Souza et al. (2013) analisam a agricultura brasileira com a utilização de um modelo de fronteira de produção estocástica. Neste modelo foram considerados como efeitos técnicos afetando a componente de eficiência a intensidade da pesquisa, a assistência técnica, efeitos regionais e a probabilidade de renda líquida negativa. A função de produção postula a presença dos insumos terra, trabalho e tecnologia. Considerando ambos os censos, a tecnologia é o fator mais importante para o crescimento da produção. De fato, para os dados de 2006 , o trabalho correspondeu a $22,3 \%$, tecnologia a $68,1 \%$ e terra a $9,6 \%$.

Como acentuam Alves et al. (2012), há um problema sério de difusão de tecnologia na agricultura brasileira. Com efeito, pelo Censo Agropecuário de 2006, o Instituto Brasileiro de Geografia e Estatística (IBGE) registrou cerca de 4,4 milhões de estabelecimentos que informaram produção e área explorada. Destes, 500 mil, 11,4\% do total, produziram $86,6 \%$ do valor da produção. No grupo de 500 mil, há 27.306 estabelecimentos que sozinhos geraram $51,2 \%$ do valor da produção de 2006. Os restantes 3,9 milhões de estabelecimentos, $88,6 \%$ do total, geraram somente $13,4 \%$ do valor da produção. Neste grupo há 2,9 milhões de estabelecimentos, $66,0 \%$ do total, que contribuíram apenas com 3,3\% do valor da produção de 2006.

Observações como essas motivam a necessidade de estudos que identifiquem regiões e áreas onde se deve orientar a pesquisa agropecuária e a assistência técnica via programas de inclusão tecnológica. É claro que tais ações não estão dissociadas de políticas públicas, notadamente aquelas de impactos sociais e econômicos. Neste contexto, como acentuam Alves e Souza (2014), é preciso ressaltar o papel do entorno ao estabelecimento na adoção de tecnologia. $\mathrm{O}$ entorno define a lucratividade da tecnologia, e sem esta lucratividade não há adoção. $\mathrm{O}$ entorno favorável dá igualdade de oportunidades à pequena e à grande produção. Quando existe esta igualdade de oportunidade, a extensão rural tem condições de ser bem sucedida com a agricultura familiar. Num ambiente de sucesso, a extensão rural tem incentivos para ser ainda melhor. Quando este inexiste por um longo tempo, o foco em difusão de tecnologia é mudado e o pessimismo domina. Há, assim, forte interação entre o entorno e a qualidade da extensão rural. Num entorno desfavorável, se a extensão limitar sua ação ao estabelecimento, certamente não será bem sucedida. Outra forma de se referir a mesmo assunto é tratar das imperfeições de mercado (Alves e Silva, 2013).

Neste artigo procura-se caracterizar a intensidade dos efeitos pesquisa, extensão e de indicadores diretamente relacionados às condições de entorno na eficiência técnica de produção agrícola municipal, agregada em nível de mesorregião. A exposição prossegue como segue. Na Seção 2 descreve-se a base de dados utilizada no estudo. Na Seção 3 descreve-se o modelo de produção utilizado e a abordagem estatística proposta para a avaliação dos fatores contextuais. Na Seção 4 apresentam-se os resultados do estudo. Finalmente, a Seção 5 apresenta um resumo e as conclusões do estudo.

\section{VARIÁVEIS DE PRODUÇÃO, FATORES CONTEXTUAIS E DADOS}

Os dados de produção utilizados neste trabalho foram obtidos dos microdados do Censo Agropecuário de 2006. Foram agregados em nível municipal. Considerou-se a renda bruta média do estabelecimento como variável resposta do modelo de produção e os gastos médios com terra, trabalho e insumos tecnológicos, por município, como fatores de produção. A Tabela 1 descreve em detalhes as componentes de valor dessas variáveis. Têm-se dados válidos para 5474 municípios, que representam 98,4\% do total de municípios brasileiros.

As variáveis contextuais consideradas no estudo são variáveis indicadoras regionais Centro-Oeste, Norte, Nordeste, Sul e Sudeste, proporção de estabelecimentos que receberam assistência técnica, impacto da pesquisa da Embrapa e um indicador de condições sociais do município. 
O impacto da pesquisa da Embrapa foi medido no nível de mesorregião. Representa uma proxy da percepção dos pesquisadores da Embrapa sobre a área e o nível de influência das tecnologias geradas pela empresa na melhoria da renda dos produtores rurais à época do Censo Agropecuário de 2006. A percepção quanto à influência da pesquisa foi classificada como baixa, média e alta. Tomou-se como covariável a proporção de percepções consideradas média e alta.

A intensidade da assistência técnica é utilizada como proxy para extensão rural. Os dados são oriundos do Censo Agropecuário de 2006.

Tabela 1: Descrição das Variáveis.

\begin{tabular}{|c|c|c|c|}
\hline Variável & Componentes & Unidade & Comentário \\
\hline Y (produção) & $\begin{array}{l}\text { Renda bruta do } \\
\text { estabelecimento }\end{array}$ & Reais & $\begin{array}{l}\text { Valor total da } \\
\text { produção agropecuária } \\
\text { do estabelecimento }\end{array}$ \\
\hline Terra & $4 \%$ do valor da terra & Reais & $\begin{array}{l}\text { Valor de aluguel da } \\
\text { terra }\end{array}$ \\
\hline Trabalho & $\begin{array}{l}\text { Salários ou outras formas } \\
\text { de compensação pagos à } \\
\text { família e aos trabalhadores } \\
\text { contratados }\end{array}$ & Reais & - \\
\hline $\begin{array}{l}\text { Capital } \\
\text { (insumos } \\
\text { tecnológicos) }\end{array}$ & $\begin{array}{l}\text { Maquinário, melhorias no } \\
\text { estabelecimento, aluguéis } \\
\text { de equipamentos, valor de } \\
\text { culturas permanentes, } \\
\text { valor dos animais, valor } \\
\text { das matas plantadas, valor } \\
\text { de sementes, valor de sal e } \\
\text { forragens, valor de } \\
\text { medicamentos, adubação e } \\
\text { pesticidas, despesas com } \\
\text { combustíveis, eletricidade, } \\
\text { armazenagem, matérias } \\
\text { primas e outros gastos }\end{array}$ & Reais & $\begin{array}{l}\text { Todos os valores de } \\
\text { estoques foram } \\
\text { depreciados à taxa de } \\
6 \% \text { ao ano num } \\
\text { período determinado } \\
\text { que varia com o tipo } \\
\text { do estoque (máquinas } \\
-15 \text { anos; matas } \\
\text { plantadas - } 20 \text { anos; } \\
\text { culturas permanentes - } \\
15 \text { anos; benfeitorias - } \\
50 \text { anos; animais - } 5 \\
\text { anos) }\end{array}$ \\
\hline
\end{tabular}

O indicador de condições sociais agrega um conjunto de variáveis que retratam o nível de bem estar social. Leva em conta os fatores: taxa de alfabetização de pessoas com 10 ou mais anos de idade; nível de pobreza absoluta; rendimento médio mensal per capita dos domicílios rurais; complementar da proporção de crianças até 5 anos de idade residentes em domicílios particulares permanentes rurais com responsável ou cônjuge analfabeto e saneamento inadequado; proporção de domicílios rurais dotados de energia elétrica no município; índice de desenvolvimento da educação básica - IDEB-2009; proporção de domicílios rurais no município com abastecimento de água através de poços, nascentes ou ligados à rede geral; índice de desempenho do SUS - IDSUS-2011. As informações utilizadas para a construção do indicador social provêm do Censo Demográfico de 2010, Censo Agropecuário de 2006, Ministério da Saúde e Instituto Nacional de Estudos e Pesquisas Educacionais - INEP. As variáveis componentes do indicador social foram transformados em grandezas adimensionais com o uso de ordenações (ranks), normalizadas pelo máximo, e agregadas por técnica multivariada como sugerida por Moreira et al. (2004). O indicador, na realidade, é uma média ponderada das variáveis transformadas com a ponderação da $i$-ésima componente igual a $R_{i}^{2} / \sum_{j} R_{j}^{2}$, sendo $R_{i}^{2}$ o coeficiente de correlação múltipla obtido da regressão da $i$-ésima componente nas demais. Um conjunto similar de variáveis está em 
consideração pelo Instituto CNA (Confederação Nacional da Agricultura, 2013) visando à construção de um indicador global de desenvolvimento rural.

\section{ABORDAGEM METODOLÓGICA}

\subsection{MOdELO DE PROdUÇÃo}

Considere-se um processo de produção composto por 5474 firmas (municípios). Cada município faz uso do vetor de insumos $\left(x_{1}, x_{2}, x_{3}\right)$ - terra, trabalho e tecnologia - para produzir o nível de produto $y$. Seja $Y=\left(y_{1}, y_{2}, \ldots, y_{5474}\right)$ o vetor produto do processo. Seja $X$ a matriz $3 \times 5474$ de uso dos insumos. A $r$-ésima coluna de $X$ é o vetor de insumos utilizados pelo município $r$.

A medida de eficiência técnica de produção $\phi^{*}\left(x_{o}, y_{o}\right)$, mais geralmente de performance, para o município $o$, com vetor de produção $\left(x_{o}, y_{o}\right)$, derivada da abordagem de Análise de Envoltória de Dados - DEA, orientada a produto e com retornos variáveis à escala, é dada por:

$$
\phi^{*}\left(x_{o}, y_{o}\right)=\max _{\phi, \lambda} \phi
$$

sujeito a
i) $Y \lambda \geq \phi y_{o}$
ii) $X \lambda \leq x_{o}$ e iii) $\lambda \geq 0, \lambda 1=1, \phi$ livre

Faz-se mister observar aqui que as variáveis de produção (produto e insumos) foram consideradas com médias por município, transformadas por ranks e normalizadas pelo máximo no cálculo da medida DEA de performance. A transformação em ranks é compatível com as transformações utilizadas nas variáveis contextuais e define uma abordagem não paramétrica que permite a estimativa da eficiência sem a influência de valores extremos no espaço das variáveis de produção. É também uma transformação usual em Estatística Não Paramétrica na presença de não normalidade, observações atípicas e heteroscedasticidade (Connover, 1999).

\subsection{AnÁlise Estatística da InfluênCIa das Covariáveis na MEdida de PERformance}

Preliminarmente as variáveis contextuais foram ajustadas para observações por mesorregião, com o objetivo de torná-las compatíveis com as informações de percepção da pesquisa. Esse ajuste foi obtido por meio do cálculo de médias. A medida de performance foi ajustada calculando-se para cada mesorregião a proporção de municípios com medida de performance superior à mediana nacional $(0,517)$. $O$ efeito na performance é estudado por meio desta variável resposta. Têm-se 129 observações válidas.

O modelo estatístico utilizado baseia-se na proposta de Ramalho et al. (2010) com o uso de regressão fracionária. Seja $\hat{\theta}_{\tau}$ a resposta da mesorregião $\tau$ relacionada à variável dependente, e $w_{\tau}$ o vetor de variáveis contextuais. Postula-se que $E\left(\hat{\theta}_{\tau} \mid w_{\tau}\right)=G\left(w_{\tau} \delta\right)$, sendo $G($.) uma função de distribuição de probabilidades. O parâmetro desconhecido $\delta$ é estimado por mínimos quadrados não lineares, procurando-se pelo mínimo de $\sum_{\tau}\left(\hat{\theta}_{\tau}-G\left(w_{\tau} \delta\right)\right)^{2}$. Escolhas competitivas para $G$ são as funções de distribuição logística, normal padrão e a inversa da distribuição do valor extremo. São dadas respectivamente por $G(u)=e^{u} /\left(1+e^{u}\right)$, $G(u)=\Phi(u)$, e $G(u)=1-e^{-e^{u}}$. A função $\Phi(u)$ é a normal padrão.

A correlação entre as mesorregiões induzida pelo cálculo da medida DEA exige uma medida robusta na estimação da matriz de variância-covariância do estimador $\hat{\delta}$. Com este 
propósito fazemos uso aqui do bootstrap não paramétrico (StataCorp, 2013) com 5000 repetições.

\section{RESULTADOS ESTATÍSTICOS}

A Figura 1 apresenta o desenho esquemático das observações de intensidade das ações de pesquisa. As percepções mais intensas caem na região Norte, seguida das regiões Sul e Sudeste. Algumas observações atípicas discordantes encontram-se na região Nordeste.

Na Figura 2 apresenta-se a distribuição, por região, do indicador de assistência técnica. As regiões Sul, Nordeste e Sudeste dominam, seguidas das regiões Centro-oeste e Norte, sugerindo variação linear inversa com o indicador da percepção oriunda da pesquisa.

A Figura 3 apresenta uma visão do indicador social por região, sugerindo ações mais intensas nas regiões Nordeste, Sul e Sudeste, seguidas das regiões Centro-oeste e Norte. A maior variabilidade é da região Nordeste.

A Figura 4 ilustra a variação conjunta da medida de eficiência considerada com as covariáveis utilizadas na regressão fracionária. Com exceção da região Sudeste, os construtos social e de assistência técnica variam na mesma direção da resposta.

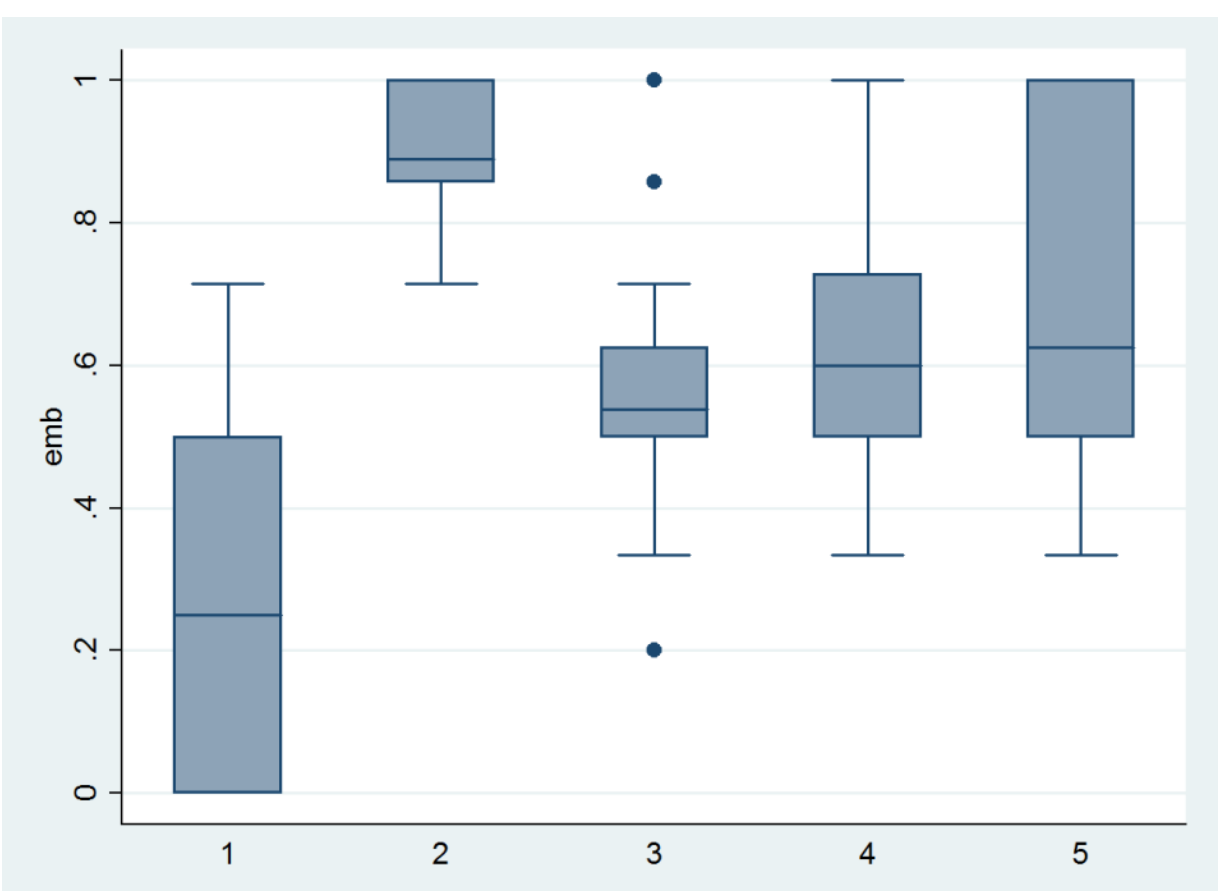

Figura 1: Desenho esquemático por região ( $1=$ Centro-oeste; $2=$ Norte; $3=$ Nordeste; $4=$ Sul; 5 = Sudeste) da proporção de concentração de pesquisa com impacto médio e alto ('bem'). 


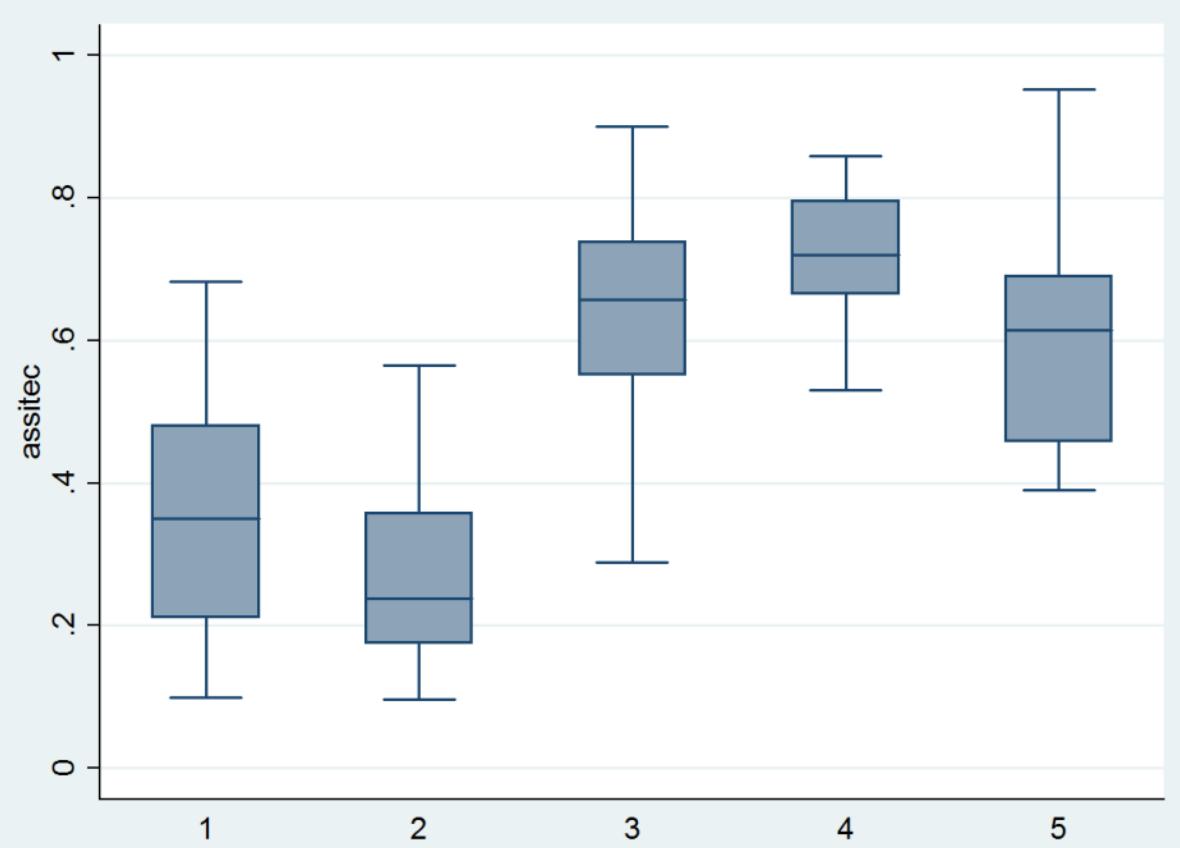

Figura 2: Desenho esquemático por região (1= Centro-oeste; 2 = Norte; 3 = Nordeste; 4 = Sul; 5 = Sudeste) do indicador de assistência técnica ('assitec').

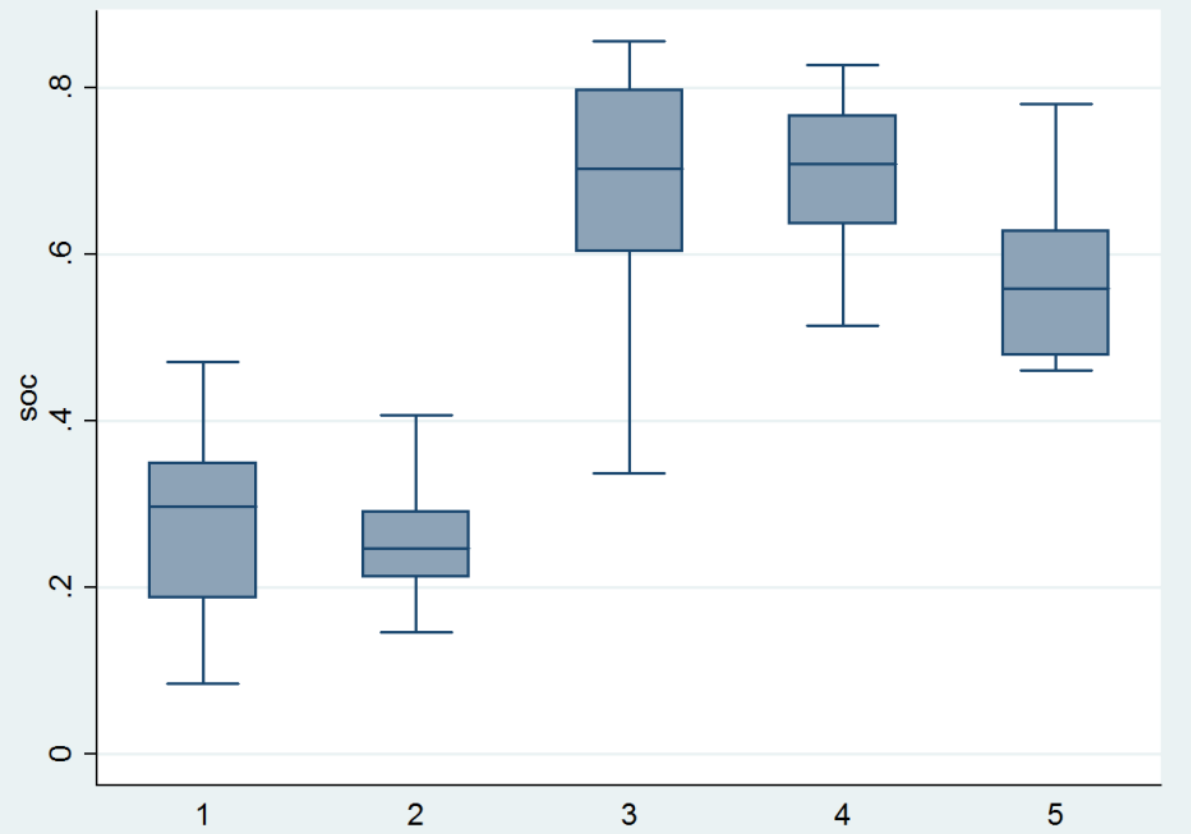

Figura 3: Desenho esquemático por região ( $1=$ Centro-oeste; 2 = Norte; 3 = Nordeste; 4 = Sul; 5 = Sudeste) do indicador social ('soc'). 


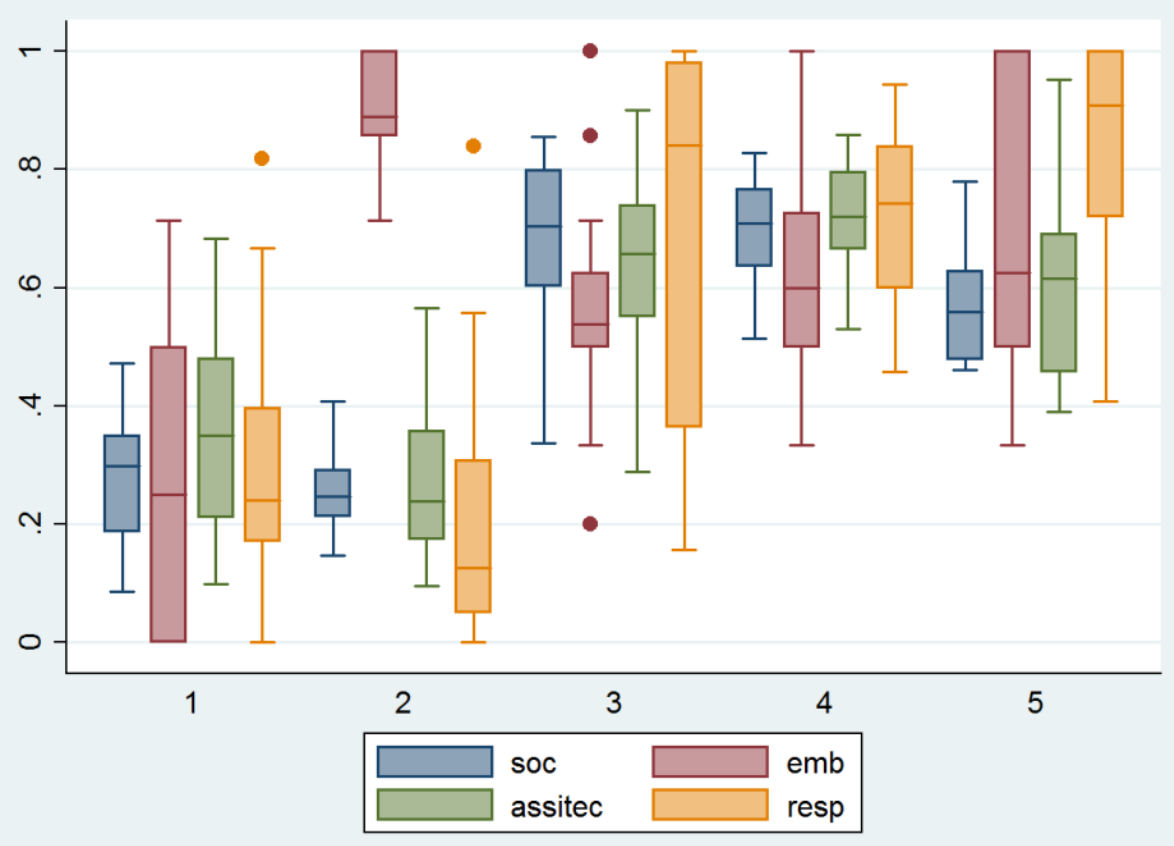

Figura 4: Desenhos esquemáticos da variável dependente e das variáveis contextuais, por região ( $1=$ Centro-oeste; $2=$ Norte; $3=$ Nordeste $;=$ Sul; $5=$ Sudeste).

A Tabela 2 apresenta os resultados obtidos com a utilização de mínimos quadrados não lineares na regressão fracionária. Os desvios-padrão reportados baseiam-se na estimativa da matriz de variância-covariância pelo método bootstrap. A forma funcional escolhida foi a inversa da distribuição do valor extremo, que apresentou menor erro médio quadrático (Tabela 3). Ressalte-se, contudo, que as três formas funcionais aqui consideradas produzem resultados análogos do ponto de vista da significância dos parâmetros.

Tabela 2: Regressão não linear da proporção de medidas DEA superiores a 0,517 na variáveis contextuais regiões, percepção da importância da pesquisa, assistência técnica/extensão rural e indicador social.

\begin{tabular}{|l|r|r|r|r|r|r|}
\cline { 2 - 7 } \multicolumn{1}{c|}{} & Coeficiente & $\begin{array}{c}\text { Desvio padrão } \\
\text { bootstrap }\end{array}$ & \multicolumn{1}{c|}{$\mathbf{z}$} & \multicolumn{2}{c|}{$\mathbf{P}>|\mathbf{z}|$} & \multicolumn{2}{c|}{$\begin{array}{c}\text { Intervalo de } \\
\text { confiança a 95\% }\end{array}$} \\
\hline Constante & $-1,5108$ & 0,469 & $-3,22$ & 0,001 & $-2,430$ & $-0,592$ \\
\hline Centro-oeste & $-0,876$ & 0,360 & $-2,43$ & 0,015 & $-1,581$ & $-0,170$ \\
\hline Norte & $-0,852$ & 0,322 & $-2,64$ & 0,008 & $-1,483$ & $-0,220$ \\
\hline Nordeste & $-0,895$ & 0,276 & $-3,25$ & 0,001 & $-1,436$ & $-0,355$ \\
\hline Sul & $-0,979$ & 0,210 & $-4,66$ & 0,000 & $-1,391$ & $-0,568$ \\
\hline Pesquisa & $-0,287$ & 0,431 & $-0,67$ & 0,505 & $-1,133$ & 0,558 \\
\hline Extensão rural & 1,243 & 0,754 & 1,65 & 0,099 & $-0,235$ & 2,7207 \\
\hline Indicador social & 2,875 & 0,981 & 2,93 & 0,003 & 0,953 & 4,798 \\
\hline
\end{tabular}

Tabela 3: Erro Médio Quadrático - Distribuições Normal, Logística e Inversa do valor extremo.

\begin{tabular}{|c|c|}
\hline Distribuição & Erro Médio Quadrático \\
\hline Normal & 0,03168 \\
\hline Logístico & 0,03178 \\
\hline Inversa do Valor Extremo & 0,03076 \\
\hline
\end{tabular}


É notório o gradiente de importância na magnitude dos efeitos. A assistência técnica tem significância positiva marginal e a pesquisa é não significativa estatisticamente. Tal resultado, do ponto de vista estatístico, segue do contexto já evidenciado nos desenhos esquemáticos, nos quais se observa variação inversa entre a percepção de impacto da pesquisa com a resposta e com as ações de assistência técnica. O impacto direto na produção agropecuária vem da extensão rural, e sua ação tem a mesma temporalidade da produção. A pesquisa cria insumos para a extensão; logo, antecede a extensão. Por isto, não se espera ser ela estatisticamente significante. Foi mantida no modelo para testar esta hipótese.

É altamente significante o efeito positivo do indicador social, evidenciando a necessidade de políticas públicas na efetividade da implementação de ações de extensão e de transferência de tecnologia. Tais resultados dão suporte quantitativo às observações de Alves e Souza (2014) e salientam a necessidade de políticas públicas especificamente voltadas para a melhoria das condições do entorno: "Tanto na Europa como nos Estados Unidos, as condições de vida no meio rural e em suas comunidades são boas, quanto à qualidade de vida. Aqui no Brasil, há muitas deficiências nas estradas rurais, em eletricidade, acesso à televisão, telefonia e qualidade da infraestrutura de saúde e educação das pequenas cidades. Estas coisas fazem parte do entorno, e sem elas fica difícil para a pequena produção deixar a pobreza para traz, além de ser muito grande o incentivo para migrar. É de justiça que se esforce para solucionar estas deficiências.".

A Figura 5 ilustra o ajuste obtido com a especificação da função inversa da distribuição do valor extremo. A correlação entre valores observados e preditos é 0,863.

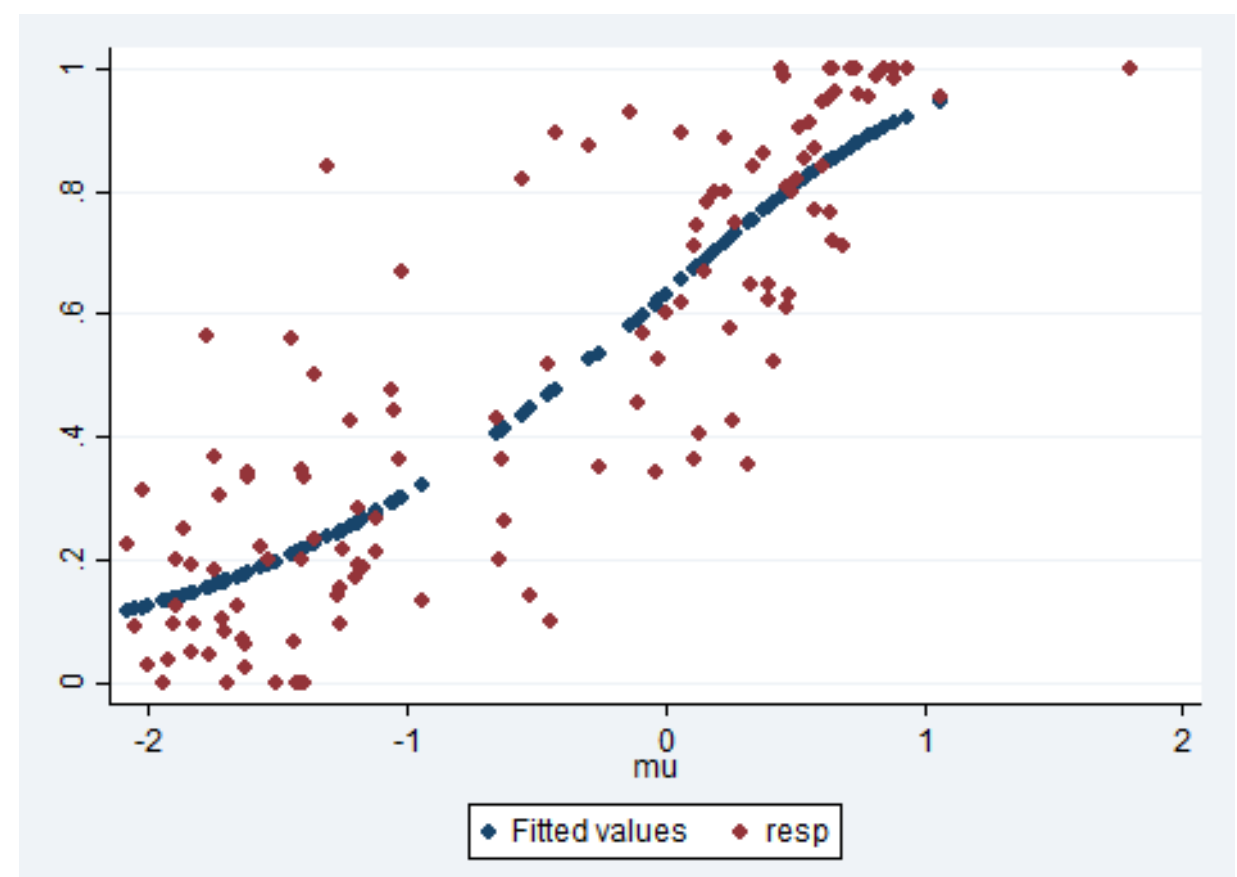

Figura 5: Valores observados ('resp') e ajustados ('Fitted values') - Regressão não linear. Inversa da distribuição do valor extremo.

O efeito marginal do indicador social é dado pela expressão $2,875 \exp \left(w_{\tau} \hat{\boldsymbol{\delta}}-\exp \left(w_{\tau} \hat{\boldsymbol{\delta}}\right)\right)$, dependente do nível das variáveis contextuais e, portanto, das mesorregiões. A Tabela 4 apresenta os valores do efeito social esperado por região, calculado nos respectivos valores médios de $w_{\tau} \hat{\delta}$. As diferenças regionais não suficientemente marcantes para garantir diferenças significantes estatisticamente, pois os intervalos de confiança se interceptam. Conforme o desenho esquemático da Figura 6, os maiores efeitos possíveis ocorrem nas regiões Sul, Nordeste e Sudeste, seguidos das regiões Centro-oeste e Norte. 
Tabela 4: Efeito social esperado por região. Intervalos de confiança com base na distribuição normal e desvios padrão bootstrap.

\begin{tabular}{|c|c|c|c|c|c|c|}
\hline \multirow[b]{2}{*}{ Centro-Oeste } & \multirow{2}{*}{$\begin{array}{r}\text { Coeficiente } \\
0,641\end{array}$} & \multirow{2}{*}{$\begin{array}{r}\text { Desvio padrão } \\
0,219\end{array}$} & \multirow{2}{*}{\begin{tabular}{l|}
$\mathbf{z}$ \\
2,93
\end{tabular}} & \multirow{2}{*}{$\begin{array}{l}\mathbf{P}>|\mathbf{z}| \\
0,003\end{array}$} & \multicolumn{2}{|c|}{$\begin{array}{c}\text { Intervalo de } \\
\text { confiança a } 95 \%\end{array}$} \\
\hline & & & & & 0,212 & 1,070 \\
\hline Norte & 0,487 & 0,166 & 2,93 & 0,003 & 0,161 & 0,812 \\
\hline Nordeste & 1,042 & 0,355 & 2,93 & 0,003 & 0,345 & 1,739 \\
\hline Sul & 1,020 & 0,348 & 2,93 & 0,003 & 0,338 & 1,702 \\
\hline Sudeste & 0,795 & 0,271 & 2,93 & 0,003 & 0,263 & 1,327 \\
\hline
\end{tabular}

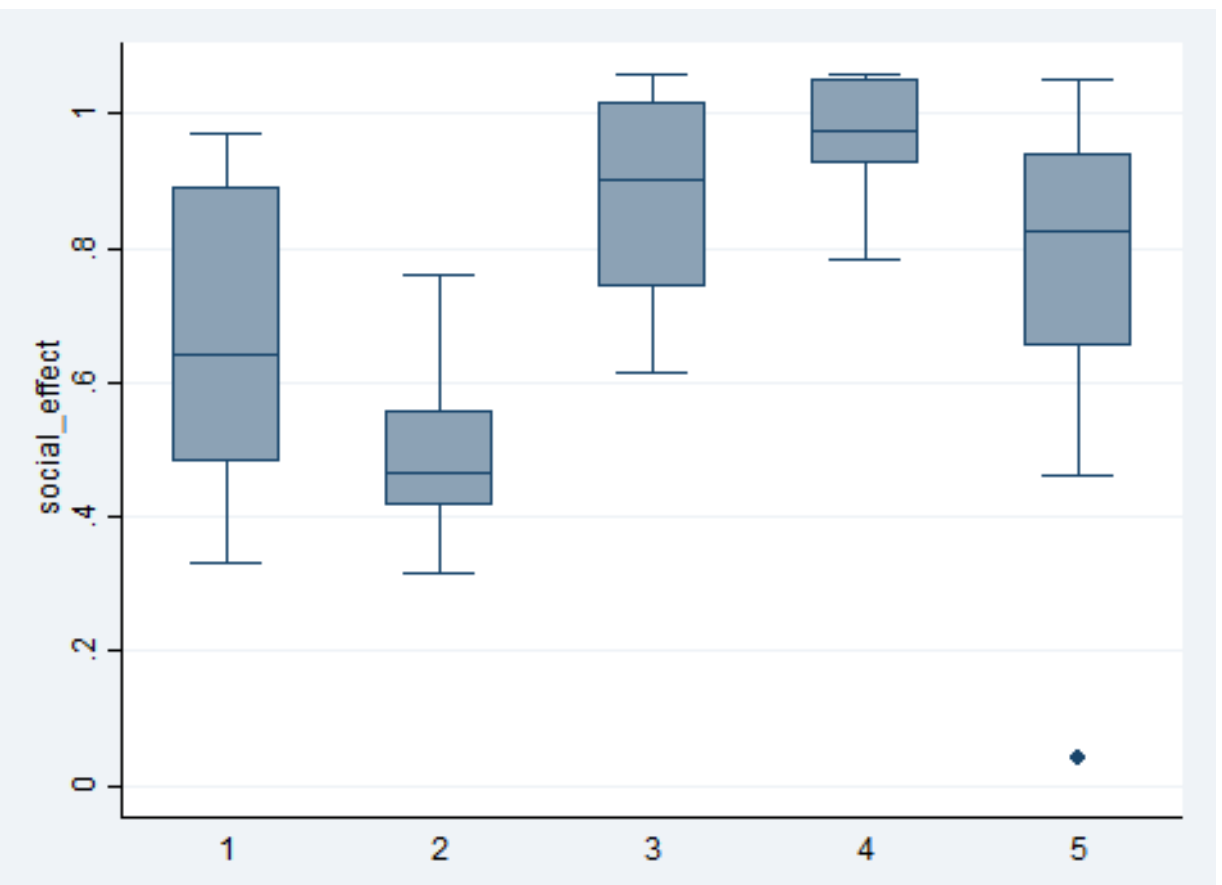

Figura 6: Desenho esquemático do efeito do indicador social ('social_effect') na resposta, por região ( $1=$ Centro-oeste; $2=$ Norte; $3=$ Nordeste; $4=$ Sul; $5=$ Sudeste).

\section{RESUMO E CONCLUSÕES}

Estudaram-se os efeitos de proxies para pesquisa, extensão e políticas públicas na medida de performance, tomando-se por base a Análise de Envoltória de Dados. Neste trabalho, no nível de mesorregião, considerou-se como resposta a proporção das melhores performances. A proxy de políticas públicas utilizada é definida por um indicador social, que inclui componentes de educação, saúde, renda e infraestrutura (água, energia e saneamento).

O gradiente de importância dos efeitos estimados indica a ordenação políticas públicas, extensão e pesquisa. A extensão rural tem papel importante e a pesquisa, como esperado, não mostrou significância estatística.

O indicador social, que capta a influência de muitas variáveis que definem as condições do entorno ao qual o estabelecimento pertence, é estatisticamente significante. Confirma isto a hipótese de que sem eliminar as imperfeições de mercado que estão entre o produtor e o consumidor, dificilmente resolver-se-á, pela agricultura, a pobreza rural que oprime milhões de agricultores. E, assim, ficar-se-á cada vez mais dependente de políticas de transferência de renda para mitigar o sofrimento de milhões de agricultores, a maioria deles nordestinos. É imperativo que, ao lado do investimento em extensão rural e pesquisa, eliminem-se as imperfeições de mercado. Caso contrário, estes investimentos não renderão o esperado para sociedade e até podem decepcioná-la, no que tange aos excluídos da modernização da agricultura. 
Mais especificamente, sugere-se a importância da melhoria dos aspectos sociais e de infraestrutura para tornar viáveis as ações de extensão rural (e indiretamente da pesquisa) que visem o incremento do desempenho na produção agropecuária. Todas as regiões seriam beneficiadas significativamente com essa política.

\section{REFERÊNCIAS}

[1] Alves, E.R.A.; Silva, R.C. Qual é o problema de transferência de tecnologia do Brasil e da Embrapa? In: Alves, E.R.A.; Souza, G.S.; Gomes, E.G. (Org.). Contribuição da Embrapa para o desenvolvimento da agricultura no Brasil, p. 182-191, 2013.

[2] Alves, E.R.A.; Souza, G.S. Desafios da Agência de Extensão Rural, 2014. Documento não publicado.

[3] Alves, E.R.A.; Souza, G.S.; Rocha, D.P. Lucratividade da Agricultura. Revista de Política Agrícola, ano XXI, n. 2, p. 45-63, 2012.

[4] Confederação Nacional da Agricultura. Índice de Desenvolvimento Rural CNA, 2013. Documento não publicado.

[5] Conover, M.J. Practical Nonparametric Statistics. 3rd ed. New York: Wiley, 1999.

[6] Moreira, T.B.; Pinto, M.B.; Souza, G.S. Uma metodologia alternativa para mensuração de pressão sobre o mercado de câmbio. Estudos Econômicos, v. 34, p. 73-99, 2004.

[7] Ramalho, E.A.; Ramalho, J.J.S.; Henriques, P.D. Fractional regression models for second stage DEA efficiency analyse. Journal of Productivity Analysis, v. 34, p. 239$255,2010$.

[8] Souza, G.S.; Alves, E.R.A.; Gomes, E.G.; Magalhães, E.; Rocha, D.P. Um modelo de produção para a agricultura brasileira e a importância da pesquisa da Embrapa. In: Alves, E.R.A.; Souza, G.S.; Gomes, E.G. (Org.), Contribuição da Embrapa para o desenvolvimento da agricultura no Brasil, p. 49-86, 2013.

[9] StataCorp. Base Reference Manual. Texas: Stata Press, 2013. 